\title{
Elbow dislocation. Review of current concepts
}

\author{
Sergio Rivera-Zarazúa, Yadira Tamez-Mata, Víctor Peña-Martínez, Gregorio Villarreal-Villarreal, \\ Mario Simental-Mendía, Ricardo Salinas-Garza, and Carlos Acosta-Olivo*
}

Department of Traumatology and Orthopedics, School of Medicine and the "Dr. Jose Eleuterio Gonzalez"University Hospital, Universidad Autónoma de Nuevo Léon, Monterrey, Nuevo Leon, Mexico

\begin{abstract}
A simple elbow dislocation represents between $51 \%$ and $74 \%$ of all elbow dislocations. It is the second joint in dislocation frequency after the shoulder. Elbow dislocations are classified as simple (without a fracture) or complex (with a fracture); direction of cubitus displacement and radius in relation to the humerus is also important. Usually, the injury mechanism indicates the dislocation type. The radiographic evaluation (gold standard) is done with an anteroposterior and lateral of the elbow joint. Treatment is related to the dislocation classification. In a simple dislocation, the objectives are a concentric, closed, and stable reduction of the joint, to gain a range of movement early. Related complications are neurological or vascular injuries, compartmental syndrome, heterotopic ossification, chronic instability, and osteoarthritis.
\end{abstract}

Key words: Elbow dislocation. Closed reduction. Fracture-dislocation. Heterotopic ossification.

\section{Anatomy and biomechanics}

The elbow joint, also known as trocleogingilomoid, has articulated mobility (ginglimoid) in flexion and extension in the humeroulnar and radiocapitellar joints (trochoid) ${ }^{1}$. It presents stability due to the congruence of the articular surface, lateral ligaments, and surrounding articular capsule. Stability is maintained by the primary static stabilizers (humeroulnar articulation, medial collateral ligament (MCL), and external collateral ligament), reinforced by secondary static stabilizers (origins of the common flexor and extensor tendons, anconeus, and triceps brachii). When they fail, the elbow becomes unstable ${ }^{2}$.

The humeroulnar joint is the cornerstone of bone stability and mobility in the elbow's flexo-extension plane. The medial portion of the coronoid plays a fundamental role in the joint stabilization in varus displacements. In extreme movements of the humerouInar joint, the olecranon or the coronoid apophysis articulate in their corresponding fossae, this way, they increase stability due to the muscle contraction and with a small contribution from the ligaments ${ }^{3}$.

The distal humerus provides the elbow's proximal articular surface, which is composed of the trochlea and capitellum. The radial head is a secondary stabilizer of the elbow. The concave surface of the radial head is articulated with the capitellum, while the border of the radial head is articulated with the lesser sigmoid notch. The articular cartilage covers the concave surface and an arch of approximately $280^{\circ}$ from the border. The radial head is not completely circular and is displaced variably from the neck axis, which is important to know in the reconstruction of the radial head ${ }^{1}$. 
The highly congruent surface of the proximal ulnar and the trochlea form one of the main limitations of elbow articulation. The sagittal crest of the greater sigmoid notch extends longitudinally and is articulated with the trochlea apex. The concavities which are medial and lateral to the sagittal crest complement the medial and lateral convex surfaces of the trochlea ${ }^{1}$.

The soft tissues play an important part in stability through the ligaments, the articular capsule, the common extensor origin, and some muscles that pass over this joint. The MCL consists of 3 bundles or bands: anterior, posterior, and transverse. The anterior band is a primary static stabilizer, which resists the stress in valgus ${ }^{4}$. The lateral ulnar collateral ligament (LUCL) is made up of the LUCL, radial collateral ligament $(\mathrm{RCL})$, and annular ligament. The LUCL gives stabilization during stress in varus and posterolateral rotation. The locus control region stabilizes the radial-capitellum and radial-ulnar articulation, while the annular ligament stabilizes the proximal ulnar-radial joint ${ }^{3,4}$. The muscles such as the common extensor origin and the flexor-pronator mass are secondary stabilizers which give stability during their contraction ${ }^{4}$.

\section{Epidemiology and classification}

The elbow is the second most frequent joint that presents dislocations, just below the shoulder ${ }^{3,5}$. Simple dislocations represent between $51 \%$ and $74 \%$ of all dislocations ${ }^{4}$. They represent between $11 \%$ and $28 \%$ of the total of elbow dislocations, with an annual incidence of 6 and 8 cases for every 100,000 inhabitants. Variants of the anatomical norm (varus or valgus ulnar) or deviations of the axis corresponding to previous accidents are predisposal factors for dislocations ${ }^{6}$. The non-dominant elbow is usually the most affected, probably as a protective instinct to protect the dominant limb?

Anakwe et al. ${ }^{8}$ reported an annual incidence of 2.9 (95\% IC, 1.8-3.5) for every 100,000 inhabitants. In 110 identified patients, $54 \%$ were men, with an average age of 38.8 years and with the following mechanisms of injury: falls from their own height $(57 \%)$, sports injuries (17\%), falls from a height higher than $1.2 \mathrm{~m}(13 \%)$, attacks/robberies $(10 \%)$, and pedestrians hit by cars $(6 \%)$. On the other hand, Stonebakc et al. reported an incident of 5.21 for every 100,000 inhabitants, observing that adolescents present a higher risk, and that close to half occur in athletes, men in football and women in gymnastics ${ }^{9}$. Their findings were similar to those observed by Gierer et al., who identified that almost $50 \%$ of dislocations occur during sporting events ${ }^{6}$.
Table 1. Classification of elbow dislocations described by Hildebrand et al. ${ }^{10}$

\begin{tabular}{|l|l|}
\hline \multicolumn{2}{c}{ Elbow dislocation } \\
\hline Simple & Complex \\
\hline $\begin{array}{l}\text { Anterior } \\
\text { Posterior } \\
\text { Lateral } \\
\text { Divergent }\end{array}$ & $\begin{array}{l}\text { Anterior } \\
\text { Posterior }\end{array}$ \\
\hline
\end{tabular}

Elbow dislocations are classified according to the absence (simple) or presence (complex) of fractures and the direction of the displacement of the radius and ulnar in respect to the humerus ${ }^{10}$ (Table 1). Simple luxations are more common than complex ones (51-74\%). Posterior or posterolateral displacements are the most common directions $(97 \%)$, while anterior dislocations are the least common (2-3\%). Dislocations, simple or complex, are the result of high-energy trauma, and associated injuries have been described in $11-15 \%$ of the cases. Associated injuries include shoulder, distal radius, and/or ulnar and carpus injuries ${ }^{10}$.

\section{Mechanism}

Usually, the type of dislocation is indicated by the mechanism or the elbow injury. Posterior dislocations are generally produced by a fall on the hand or wrist. The force of the fall is transmitted to the elbow in extension, causing a force that displaces the ulnar outside of the trochlea. Since the joint remains hyperextended, the anterior capsule and collateral ligaments undergo an increasing tension force, which eventually causes them to break. In addition to the hyperextension, an overload in the valgus is produced at a moment of posterolateral external rotation ${ }^{3}$. Corpse studies have proven that the extension and a moment in varus linked with elbow luxation first interrupt the LUCL ${ }^{11}$. Anterior dislocations may be caused by a direct trauma over the posterior surface of the forearm, in a position of light flexion ${ }^{3}$.

For a dislocation to occur, three stages of elbow instability ought to happen. The first stage involves only the disruption of the ulnar component of the lateral collateral ligament with a posterolateral subluxation, and the reduction is spontaneous. The second stage occurs with a progressive disruption, anterior as well as posterior, combined with an incomplete posterolateral dislocation. The third stage is divided into the $3 \mathrm{~A}$, where the anterior band of the least common multiple remains 
intact, and the 3B, where all soft tissues become altered, causing a complete luxation ${ }^{10}$.

\section{Physical evaluation}

Elbow dislocation is an emergency; therefore, the patient should go to an orthopedist immediately. The evaluation should start with a complete clinical history, questioning the mechanism of the injury, as well as the associated injuries present ${ }^{12}$. The patients typically arrive with the elbow flexed in a $45^{\circ}$ angle, with evident deformity and pain, shortening of the extremity and limitation in the range of motion (ROM). In the case of an anterior dislocation, the elbow is completely extended. Some patients may perform an auto-reduction or present spontaneous reduction and only present pain, edema, and ecchymosis.

When a $90^{\circ}$ flexion is requested, the medial and lateral epicondyles and the olecranon process should form an isosceles triangle, if they do not, it is possible that the elbow is luxated or subluxated. It is important to identify if there are superficial or deep wounds, in addition to conducting a neurological exam to assess the integrity of motor and sensorial functions. The ulnar nerve is the one affected most frequently; however, the median and radial nerves also should be assessed ${ }^{13}$. During a peripheral neurological examination, the adduction of the thumb or fingers ought to be evaluated, as well as the abduction of said digits to identify the integrity of the ulnar nerve. The extension of the thumb or wrist should be requested to assess the radial nerve and the median nerve should be evaluated through the opposition of the thumb ${ }^{14}$. Vascular examination should include a comparison of radial and ulnar pulses. It is expected that both (injury vs. collateral) be equal. If a decrease is identified, the arm-arm indexes are useful to determine if there is a vascular injury. Reports suggest that vascular injuries occur in $0.3-6 \%$ of closed dislocations and $33 \%$ of open ones ${ }^{13}$. The diagnosis of elbow dislocation is clinical and confirmed through anteroposterior (AP) and lateral X-rays $\mathbf{s}^{3,5}$. The shoulder should be included in the patient examination, as well as the ipsilateral wrist since they can be injured. The increase in localized volume due to flow interruption and the injury of soft tissue is common, and it may lead to constriction of the muscle compartments, originating the development of a compartmental syndrome ${ }^{15}$.

\section{Image studies}

Vinson et al. identified that if the patient presents normal ranges of movement or ROM (flexion, extension, pronation, and supination), X-rays could be

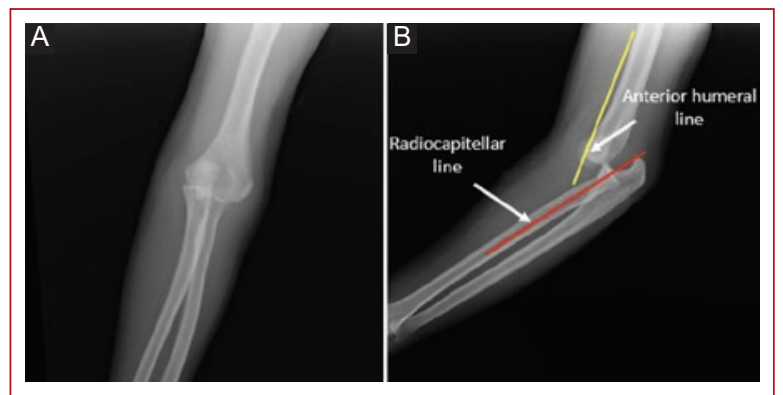

Figure 1. Dislocation of the right elbow of a 27-year-old male patient. Initial radiographs in projections.

A: anteroposterior. B: lateral, showing a loss of the anterior humeral line (yellow line) as well as the radiocapitellar. Line (red line) indicated with the white arrows, indicating simple posteromedial dislocation of the elbow. Initial management involved reduction with external maneuvers.

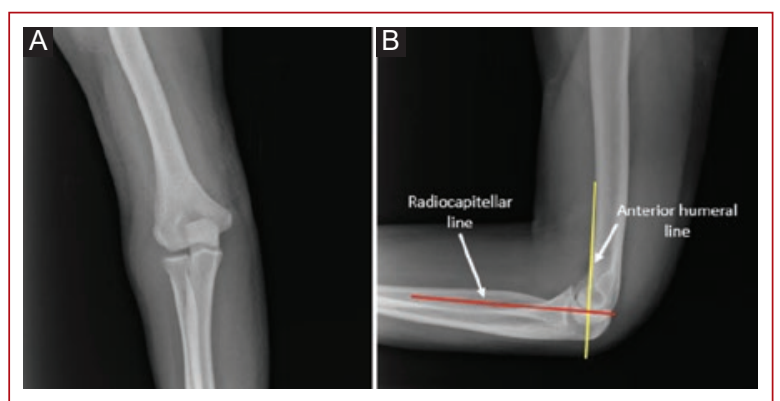

Figure 2. After reduction with external maneuvers radiographs in projections. A: radiograph anteroposterior. B: radiograph lateral in which a congruence of the anterior humeral line (yellow line) as well as the radiocapitellar line (red line) are observed and indicated by the white arrows.

omitted. They concluded that the ROM evaluation has a sensitivity of $99 \%$ to rule out injuries, and $100 \%$ sensitivity to identify injuries that require surgical intervention ${ }^{16}$. However, imaging studies are suggested to patients with acute trauma and who present the inability to flex the elbow. X-rays in AP and lateral projections are the gold standard. Other studies highlight the need to include additional studies such as oblique projections and the visualization of the radial head to complement the diagnostic evaluation ${ }^{3,5,12}$

During sporting events, some authors recommend an immediate reduction ${ }^{17}$, while other authors recommend $X$-rays before executing any kind of maneuver, to identify associated fractures or injuries and assess the articular congruence ${ }^{13}$.

AP projection (Figs. $1 A$ and $2 A$ ) is performed with the elbow in extension with the forearm in a supine 


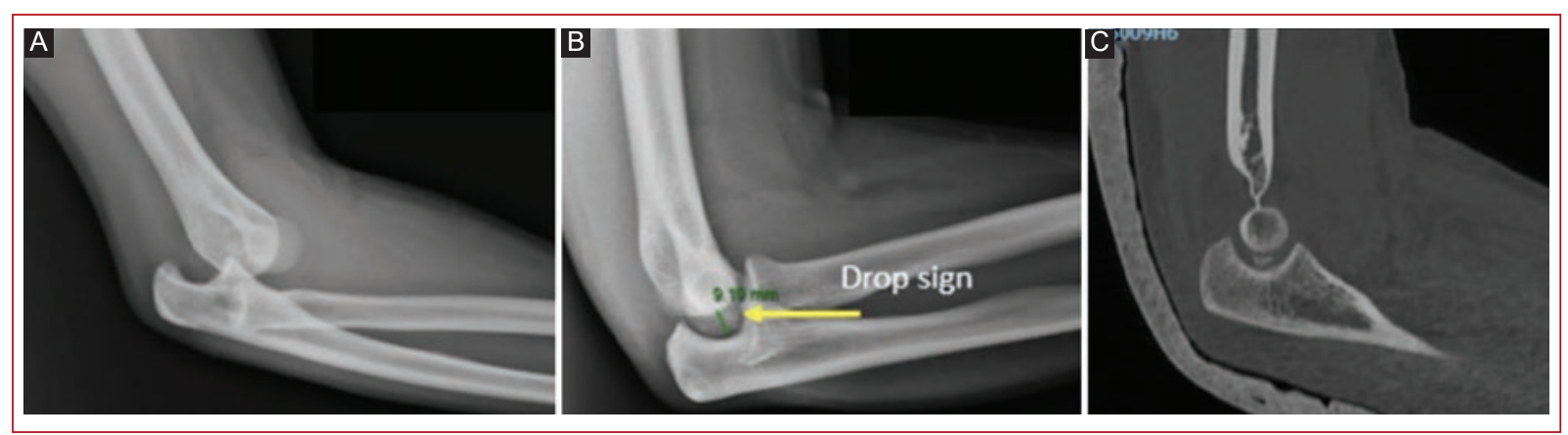

Figure 3. Posterior dislocation of the elbow on the right side of a 40-year-old male patient. A: initial lateral X-ray. B: lateral X-ray after reduction by external maneuvers, in which an ulnar humerus distance of $9.19 \mathrm{~mm}$ is observed, with the sign of the fall or drop sign indicated by the yellow arrow being positive. C: sagittal computed tomography scan of the elbow, showing the intra-articular bone fragment.

position. If the elbow cannot be fully extended due to pain or an increase in volume, the image will come out with superposition of the radial head and capitellum, causing an inadequate image ${ }^{5}$. Lateral projection (Figs. 1B and 2B) is conducted with the elbow flexed at $90^{\circ}$. Alignment may be evaluated through the anterior humeral line and the radial capitellar line. The anterior humeral line is drawn downwards of the anterior face of the humerus and should intersect the mid-third of the capitellum. The radial capitellar line is traced along the longitudinal axis of the radius and should go through the capitellum. These lines are used in both adults and pediatric patients ${ }^{5}$. Moreover, the drop sign should be evaluated (Fig. 3), which is the ulnar-humeral distance that is measured from the trochlear groove up to the olecranon. If it is $\geq 4 \mathrm{~mm}$, it is positive (+) and suggests articular instability ${ }^{5,13}$. In this same projection, we are able to assess the fat pads to identify hidden fractures. The anterior fat pad is usually seen in the lateral view as a radiolucent line which is close to the coronoid fossa. The posterior fat pad is not usually seen, and when present, it is always pathological. An articular leak of hematologic origin will displace and elevate the anterior fat pad, producing the sail sign (Fig. 4). Similarly, the posterior fat pad will rise out of the olecranon fossa and will be visualized as an articular leakage ${ }^{5}$.

Computerized axial tomography is another tool used to identify hidden injuries like fractures since the identification of these would improve management. In any case, it is not required as routine ${ }^{5}$.

Magnetic resonance imaging is another tool which may be necessary when following up on complex injuries or those difficult to diagnose; however, it is not a part of initial management. It is indicated if the patient

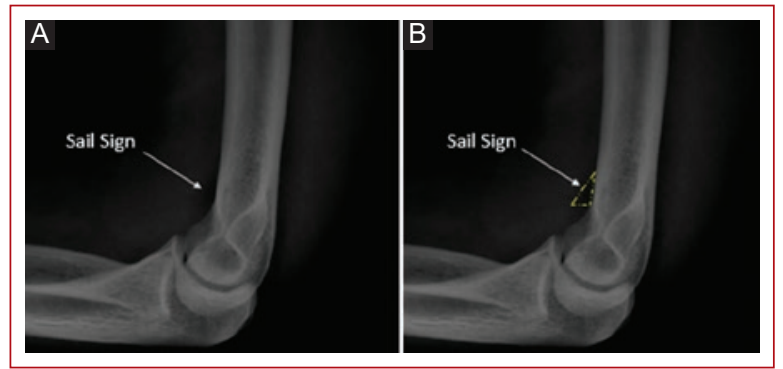

Figure 4. Dislocation of the right elbow of a 27 -year-old male patient. After reduction with external maneuvers, it was observed in the lateral radiograph. A: radiolucency in the anterior cortex of the distal humerus, indicating joint effusion (in this case of hematological origin) displacing and elevating the anterior fat pad, showing the sail sign (marked with a white arrow). B: sail sign highlighted with the yellow dotted triangle.

continues with instability during the physical examination after 2 or 3 weeks or has recurrent dislocations. It should also be considered in symptomatic cases which are unable to comply with physical examination ${ }^{5,13}$.

\section{Treatment}

Elbow dislocation classification will determine its management. In a simple dislocation, the main objective is to perform a stable concentric closed reduction, one which allows early mobilization of the active ROMs. In general, most simple dislocations are treated conservatively. This can be performed under anesthesia and sedation, or under complete sedation in an operating room. Reduction involves flexion of $25^{\circ}$ or $30^{\circ}$ combined with supination of the forearm and resistance or traction of the arm by the assistant. It is described 


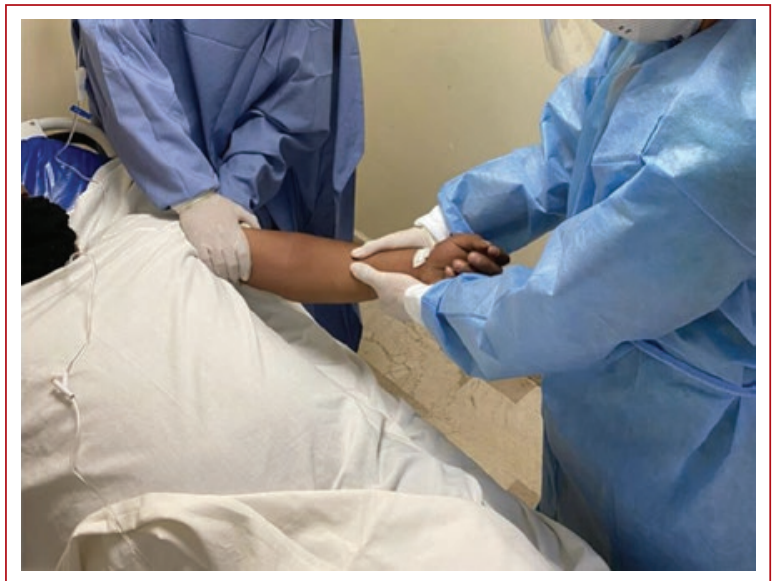

Figure 5. Traction-contraction maneuver performed on a patient with a posteromedial dislocation of the left elbow.

as a "clink" sound at the moment of reduction, which suggests a stable reduction $3,18,19$.

Conservative management is indicated if there is: (a) post-reduction stability and (b) concentric elbow reduction, including cases with the drop sign ${ }^{4,20}$.

Reduction of a posterior elbow luxation may be performed using different techniques. The first and most commonly used is traction-contraction (Fig. 5). This maneuver requires two people. Before attempting reduction, the forearm should be supinated and the elbow at a flexion of $30^{\circ}$, to decouple the coronoid apophysis and relax the bicep tendon. While the orthopedist provides stabilization of the humerus, the second assistant applies a continuous and slow longitudinal traction, which should reduce the elbow. The second assistant can also apply downward pressure over the olecranon if necessary ${ }^{3,5,12}$. Another technique, the modified Stimson maneuver (Fig. 6) can also be employed. Here, the patient is placed in the prone position with the abducted shoulder and the injured elbow on the edge of the stretcher and the forearm pointed toward the floor. The orthopedist applies traction longitudinal to the forearm while manipulating the olecranon process with the other hand. Alternatively, weight can be added to the wrist instead of downward traction. The physician should be careful concerning the position of the patient (prone) since if he is seated, this is a disadvantage in case the patient develops some emergency in the airways ${ }^{5,21}$. However, Khan et al. reported that this is an atraumatic and mechanically simple technique, which offers an anatomically congruent reduction; it is very useful in the emergency department ${ }^{22}$. Another therapeutic option is

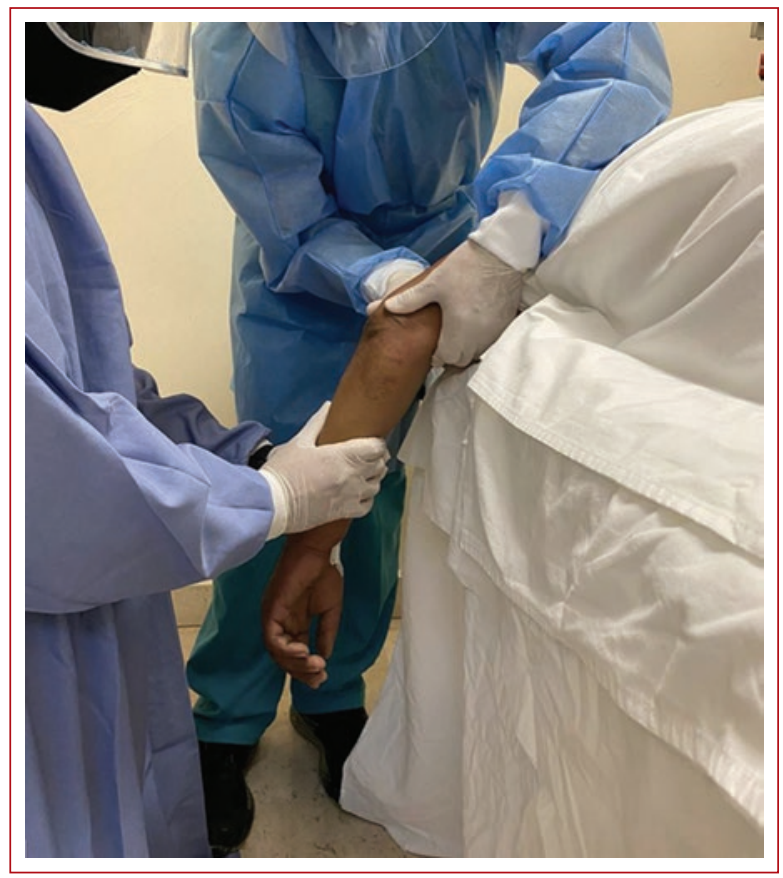

Figure 6. Modified Stimson maneuver performed in the prone position.

the leverage technique, which requires only one expert ${ }^{3,5,12}$. Moreover, one more technique to reduce posterior elbow dislocations is the Kumar technique, whose main characteristic is the soft decoupling of the coronoid process (coronoid apophysis) from the distal humerus and control over the olecranon during reduction. There is less probability of soft tissue damage when avoiding traction and hyperextension. This technique is safe, simple, and easy to learn ${ }^{5,12,23}$.

Reduction for an anterior dislocation is performed by flexing the elbow and applying downward strength over the proximal forearm instead of the distal humerus ${ }^{12,21}$. Or a traction maneuver can be performed on the wrist and countertraction on the arm, with a slight flexion and posterior displacement of the forearm ${ }^{24}$.

Once the reduction has been conducted, independently from the direction in which the displacement occurred, vascular and neurological integrity must be checked again (before and after the reduction). Ulnar neuropraxia occurs in $20 \%$ of cases and is managed conservatively. The ROM ought to be evaluated to identify articular stability. The X-rays post-reduction is necessary; as previously mentioned, the drop sign denotes an instability ${ }^{25}$; hence, adequate stability should be corroborated after reduction. Recurrent instability of $1-2 \%$ has been reported ${ }^{5}$. 
After reduction, the risk of developing compartmental syndrome should be evaluated for a period of 3 or $4 \mathrm{~h}$. If posterior evaluations are found to be without neurovascular compromises, instability or compartmental syndrome, then the patient can be discharged ${ }^{5}$.

Posterior to reduction, immobilization of the elbow joint is required. However, immobilization time has been widely debated. Hanlon et al. conducted a current revision of elbow and forearm injuries, pointing out an ideal immobilization of a week. Immobilization of more than 3 weeks showed poor results 5 .

The objective is for the patient to regain an active range of movement close to normal ranges in 6-8 weeks after the injury. About $95 \%$ of patients are able to return to their normal activities, as they did before the injury ${ }^{26}$. Rehabilitation involves resuming articular mobility; in cases where the reduction was stable, mobility should be restarted after 7-10 days since a flexion contracture can appear if early exercises are not performed. Subsequently, a sling can be utilized for the following 2-3 weeks for the patient's comfort. In cases where the reduction was unstable, a program of flexo-extension with a graduated splint can be used, with the forearm in pronation over 4 weeks ${ }^{4,11}$. In some cases, a residual subluxation has been described, occurring in 15-35\% of cases independently of previous treatment (conservative or surgical). In these cases, rehabilitation with active ROM exercises can be employed, avoiding shoulder abduction (stress in varus). This was described by Duckworth et al., who reported a successful stable elbow in 23 patients with residual subluxation, of which $5(22 \%)$ patients were previously treated conservatively and $18(78 \%)$ surgically ${ }^{20}$.

\section{Complications}

Complications which can occur in elbow dislocations can be classified according to their time of appearance, as acute (early) or chronic (late).

\section{Early}

Early complications involve neurological and vascular injuries, as well as a compartmental syndrome. The risk of a neurological injury is $1.4-29 \%$, and the nerve most commonly affected is the ulnar ${ }^{21}$. Linscheid and Wheele identified $24(22 \%)$ nervous injuries in 110 patients with elbow dislocations, of which $16(66 \%)$ were of the ulnar nerve, $3(12 \%)$ of the median nerve, and $4(16 \%)$ cases presented a mixed injury of the ulnar and median nerves ${ }^{27}$. Ulnar nerve dysfunction is usually resolved with conservative management. However, it is important to document, both pre-reduction and post-reduction, in the clinical file ${ }^{7}$.

Vascular injuries have been reported in $1-13 \%$ of elbow dislocations ${ }^{7,21}$. In addition, it has been described that between 10 and $25 \%$ maintain a radial pulse; therefore, it should be monitored and complementary studies should be performed in case of suspicion of vascular injury ${ }^{21}$.

Compartmental syndrome is a consequence of extensive soft tissue inflammation. It is more common in high-energy injuries and divergent dislocations and is associated with vascular injuries. If a diagnostic suspicion exists, inpatient admission may be warranted for observation and monitoring of compartment pressure in the affected limb. If not diagnosed, ischemia can cause muscle necrosis, and Volkomann ischemic contracture can occur. This is an ischemic contracture in the forearm, which presents with a claw-like attitude of the hand as a consequence of fibrosis that follows ischemic necrosis of the flexor muscles of the fingers and, frequently, also of the pronator muscles, as well as nervous ischemia ${ }^{7,21}$.

\section{Late}

Late complications occur after 30 days, depending on the complication. These include heterotopic ossification ( $\mathrm{HO})$, chronic instability, and osteoarthritis. $\mathrm{HO}$ has been reported in more than $75-80 \%$ of cases, related to high-energy mechanisms. However, a limitation in joint mobility has been observed in only $<5 \%$, 21 . In simple elbow dislocations, an incidence of $3 \%$ has been identified ${ }^{21}$. Shukla et al. studied 28 cases of complex elbow dislocations, observing the development of $\mathrm{HO}$ in $12(43 \%)$ of them, of which $9(75 \%)$ required multiple (two or more) attempts at closed reduction; no risk factors were found based on age or gender ${ }^{28}$.

Chronic instability has been identified in $<50 \%$ of cases. Josefsson et al. studied 52 patients with elbow dislocation in the long-term, of which $8(15 \%)$ presented chronic instability ${ }^{29}$.

Osteoarthritis has an incidence of about $80 \%$ and occurs more frequently in complex dislocations ${ }^{21}$. Josefsson et al. reported signs of degenerative joint disease in $19(36.5 \%)$ of 52 patients with elbow dislocations ${ }^{29}$. Of which $5(26 \%)$ presented sclerosis, 10 (53\%) developed osteophytes, and 14 (74\%) presented irregularities in the subchondral bone. 


\section{In summary}

- Simple elbow dislocation is a common elbow injury in $11-28 \%$ of injuries

- The most common type of simple dislocation is posteromedial, in up to $95 \%$ of cases.

- The diagnosis is clinical and is confirmed by radiographs in two views (AP and lateral).

- Posterior dislocation of the elbow can be reduced by various maneuvers, the most commonly used being traction-countertraction and modified Stimson.

- Elbow dislocations are not without complications and can be acute or chronic.

After the elbow reduction, the joint is immobilized with a sling or splint for 7-10 days, and then rehabilitation exercises are started.

\section{Conflicts of interest}

The authors declare not having any conflicts of interest.

\section{Ethical disclosures}

Protection of human and animal subjects. The authors declare that the procedures followed were in accordance with the regulations of the relevant clinical research ethics committee and with those of the Code of Ethics of the World Medical Association (Declaration of Helsinki).

Confidentiality of data. The authors declare that they have followed the protocols of their work center on the publication of patient data.

Right to privacy and informed consent. The authors have obtained the written informed consent of the patients or subjects mentioned in the article. The corresponding author is in possession of this document.

\section{References}

1. Bryce $C D$, Armstrong $A D$. Anatomy and biomechanics of the elbow. Orthop Clin North Am. 2008;39:141-54.
2. White TO, Mckenzie SP, Gray AJ. Orthopaedic Trauma. $3^{\text {rd }}$ ed. Poland: Elseiver; 2016. p. 204-7.

3. Court-Brown CM, Heckman JD. Fracturas en Adultos. $8^{\text {th }}$ ed. China: Michael McKee; 2015. p. 1179-90.

4. Slutsky DJ. Operative Orthopedics of the Upper Extremity. $1^{\text {st }}$ ed. United States: McGraw-Hill Education; 2014. p. 493-99.

5. Hanlon DP, Mavrophilipos V. The emergent evaluation and treatment of elbow and forearm injuries. Emerg Med Clin North Am. 2020;38:81-102.

6. Gierer P, Jeffries J, Herlyn P, Kittner T. Diagnosis of upper arm, elbow, and proximal lower arm fractures. Radiologe. 2020;60(7):581-590.

7. Carter SJ, Germann CA, Dacus AA, Sweeney TW, Perron AD. Orthopedic pitfalls in the ED: neurovascular injury associated with posterior elbow dislocations. Am J Emerg Med. 2010;28:960-5.

8. Anakwe RE, Middleton SD, Jenkins PJ, McQueen MM, Court-Brown CM Patient-reported outcomes after simple dislocation of the elbow. J Bone Joint Surg Am. 2011;93:1220-6.

9. Stoneback JW, Owens BD, Sykes J, Athwal GS, Pointer L, Wolf JM. Incidence of elbow dislocations in the United States population. J Bone Joint Surg Am. 2012;94:240-5.

10. Hildebrand KA, Patterson SD, King GJ. Acute elbow dislocations: simple and complex. Orthop Clin North Am. 1999;30:63-79.

11. Hobgood ER, Khan SO, Field LD. Acute dislocations of the adult elbow. Hand Clin. 2008;24:1-7.

12. Sheps DM, Hildebrand KA, Boorman RS. Simple dislocations of the elbow: evaluation and treatment. Hand Clin. 2004;20:389-404.

13. Morris MS, Ozer K. Elbow dislocations in contact sports. Hand Clin. 2017;33:63-72.

14. Hoppenfeld S. Exploración Física de la Columna Vertebral y las Extremidades. $23^{\text {th }}$ ed. México: El Manual Moderno; 1999. p. 163-70.

15. Jungbluth $\mathrm{P}$, Hakimi M, Linhart W, Windolf J. Current concepts: simple and complex elbow dislocations-acute and definitive treatment. Eur $\mathrm{J}$ Trauma Emerg Surg. 2008;34:120-30.

16. Vinson DR, Kann GS, Gaona SD, Panacek EA. Performance of the 4-way range of motion test for radiographic injuries after blunt elbow trauma. Am J Emerg Med. 2016;34:235-9.

17. Skelley NW, McCormick JJ, Smith MV. In-game management of common joint dislocations. Sports Health. 2014:6:246-55.

18. Modi CS, Lawrence E, Lawrence TM. Elbow instability. Orthop Trauma. 2012;26:316-27

19. Ahmed I, Mistry J. The management of acute and chronic elbow instability. Orthop Clin North Am. 2015;46:271-80.

20. Duckworth AD, Kulijdian A, McKee MD, Ring D. Residual subluxation of the elbow after dislocation or fracture-dislocation: treatment with active elbow exercises and avoidance of varus stress. J Shoulder Elbow Surg. 2008; $17: 276-80$

21. Gottlieb M, Schiebout J. Elbow dislocations in the emergency department: a review of reduction techniques. J Emerg Med. 2018:54:849-54.

22. Khan SK, Chopra R, Chakravarty D. Successful closed manipulation of a pure lateral traumatic dislocation of the elbow joint using a modified Stimson's technique: a case report. J Med Case Rep. 2008;2:170.

23. Kumar A, Ahmed M. Closed reduction of posterior dislocation of the elbow: a simple technique. J Orthop Trauma. 1999;13:58-9.

24. Kailash S, Shanmuganathan S. Anterior dislocation of elbow with neurovascular injury: a rare case report. J Orthop Case Rep. 2017;7:91-4

25. Sheehan SE, Dyer GS, Sodickson AD, Patel KI, Khurana B. Traumatic elbow injuries: what the orthopedic surgeon wants to know. Radiographics. 2013;33:869-88

26. Armstrong A. Simple elbow dislocation. Hand Clin. 2015;31:521-31.

27. Linscheid RL, Wheeler DK. Elbow dislocations. JAMA. 1965;194:1171-6.

28. Shukla DR, Pillai G, McAnany S, Hausman M, Parsons BO. Heterotopic ossification formation after fracture-dislocations of the elbow. J Shoulder Elbow Surg. 2015;24:333-8.

29. Josefsson PO, Johnell O, Gentz CF. Long-term sequelae of simple dislocation of the elbow. J Bone Joint Surg Am. 1984;66:927-30. 